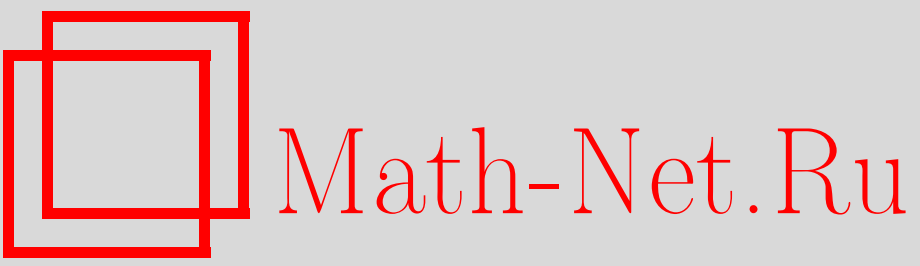

D. Ferger, D. Vogel, Weak convergence of the empirical process and the rescaled empirical distribution function in the Skorokhod product space, Теория вероятн. и ее примен., 2009, том 54, выпуск 4, 750-770

DOI: https://doi.org/10.4213/tvp3538

Использование Общероссийского математического портала Math-Net.Ru подразумевает, что вы прочитали и согласны с пользовательским соглашением

http://www. mathnet.ru/rus/agreement

Параметры загрузки:

IP: 3.85 .7 .115

26 апреля 2023 г., 13:41:22 


\title{
WEAK CONVERGENCE OF THE EMPIRICAL PROCESS AND THE RESCALED EMPIRICAL DISTRIBUTION FUNCTION IN THE SKOROKHOD PRODUCT SPACE ${ }^{1)}$
}

\begin{abstract}
Доказывается асимптотическая независимость эмпирического процесса $\alpha_{n}=\sqrt{n}\left(\mathbb{F}_{n}-F\right)$ и масштабированной эмпирической функции распределения $\beta_{n}=n\left(\mathbb{F}_{n}\left(\tau+\frac{\dot{n}}{n}\right)-\mathbb{F}_{n}(\tau)\right)$, где $F$ - произвольная непрерывная функция распределения, дифференцируемая в некоторой точке $\tau$, а $\mathbb{F}_{n}$ - соответствующая эмпирическая функция распределения. Этот результат кажется противоречащим интуиции, поскольку для любого $n \in \mathbf{N}$ существует детерминированное соответствие между $\alpha_{n}$ и $\beta_{n}$.

Точнее, показывается, что пара $\left(\alpha_{n}, \beta_{n}\right)$ сходится по распределению к пределу с независимыми компонентами, а именно к броуновскому мосту с заменой времени и двустороннему процессу Пуассона. Поскольку последние процессы имеют скачки, в частности если сама $F$ имеет скачки, то пространство Скорохода $D(\mathbf{R}) \times D(\mathbf{R})$ более всего подходит для моделирования этой сходимости. Мы развиваем теорию сходимости для $D(\mathbf{R}) \times D(\mathbf{R})$, доказывая классический принцип, разработанный Ю.В. Прохоровым, гласящий, что сходимость конечномерных распределений и плотность влекут за собою слабую сходимость. Приводится несколько критериев плотности. Наконец, сходимость пары $\left(\alpha_{n}, \beta_{n}\right)$ влечет сходимость каждой из компонент, таким образом, мы попутно даем исчерпывающее доказательство этих хорошо известных результатов о сходимости в довольно общей постановке. В действительности условие дифференцируемости $F$ хотя бы в одной точке требуется только для сходимости $\beta_{n}$ и может быть ослаблено.

Ключевые слова и фразы: топология Скорохода, броуновский мост, пуассоновский процесс, плотность семейства вероятностных мер, конечномерные распределения.
\end{abstract}

1. Introduction. This paper brings together two important convergence results in empirical process theory. The first one is the convergence in law of the uniform empirical process $\sqrt{n}\left(\mathbb{G}_{n}(t)-t\right), t \in[0,1]$, to the

${ }^{*}$ Institut für Mathematische Stochastik, Technische Universität Dresden, 01062 Dresden, Germany; e-mail: dietmar.ferger@tu-dresden.de

**Fakultät Statistik, Technische Universität Dortmund, 44221 Dortmund, Germany; e-mail: daniel.vogel@tu-dortmund.de; supported by the Collaborative Research Center (SFB) 475 of the German Research Foundation (DFG) 
Brownian bridge. Here $\mathbb{G}_{n}$ denotes the uniform empirical distribution function. This result is originally due to Donsker [7], who carried out an idea by Doob [8]. The work was motivated by the pioneer papers of Kolmogorov [15] and Smirnov [23] about the limit distribution of $\sup _{t \in[0,1]}\left|\sqrt{n}\left(\mathbb{G}_{n}(t)-t\right)\right|$ and $\sup _{t \in[0,1]} \sqrt{n}\left(\mathbb{G}_{n}(t)-t\right)$, respectively.

The other result is the convergence of the rescaled uniform empirical distribution function $n \mathbb{G}_{n}(t / n), t \geqslant 0$, to the Poisson process having intensity 1. Although this result is nowadays a standard exercise in empirical process theory, its origin has remained unknown to us. It appears in different levels of generality, e.g., in [14], [1] or [4].

The Brownian bridge, closely linked to the Brownian motion, and the Poisson process are two fundamental stochastic processes, the relevance of which goes far beyond being limit processes in asymptotic statistics. The empirical distribution function and derived processes (such as the empirical process) are an important field of study in mathematical statistics. See, for example, [25] or [26] for a profound treatment of up-to-date empirical process theory with particular focus on statistical applications.

The aim of this paper is to prove the asymptotic independence of the uniform empirical process and the rescaled uniform empirical distribution function, but we are going to do so in a general setting. Instead of being uniformly distributed, we let the underlying sequence of i.i.d. random variables $\left\{X_{n}\right\}$ be sampled from an arbitrary distribution function $F$. Then we look at the following generalizations of the uniform empirical process and rescaled uniform empirical distribution function, respectively:

$$
\begin{aligned}
& \alpha_{n}^{F}(t)=\sqrt{n}\left(\mathbb{F}_{n}(t)-F(t)\right), \quad t \in \mathbf{R}, \\
& \beta_{n}^{F, \tau}(t)= \begin{cases}n\left[\mathbb{F}_{n}\left(\tau+\frac{t}{n}\right)-\mathbb{F}_{n}(\tau)\right], & \text { if } t \geqslant 0, \\
n\left[\mathbb{F}_{n}\left(\tau+\frac{t}{n}\right)-\mathbb{F}_{n}(\tau-)\right], & \text { if } t<0,\end{cases}
\end{aligned}
$$

where $\tau$ is an arbitrary real constant and

$$
\mathbb{F}_{n}(t)=\frac{1}{n} \sum_{k=1}^{n} \mathbb{I}_{\left\{X_{k} \leqslant t\right\}}, \quad t \in \mathbf{R}
$$

is the empirical distribution function corresponding to $F$. The processes $\alpha_{n}^{F}$ and $\beta_{n}^{F, \tau}$ both converge in law to limits, say $B_{1}$ and $N_{0}$, respectively, that will be properly specified in Section 2 . We are going to show that also

$$
\left(\alpha_{n}^{F}, \beta_{n}^{F, \tau}\right) \stackrel{\mathscr{L}}{\longrightarrow}\left(B_{1}, N_{0}\right),
$$

where $B_{1}$ and $N_{0}$ are stochastically independent. 
At this point we would like to spare a few words about the implications of (1). It is quite a remarkable result. The fact that the convergence extends from the individual sequences to the joint sequence is, although not to be taken for granted, hardly surprising. But $B_{1}$ and $N_{0}$ in (1) are independent, while $\alpha_{n}^{F}$ and $\beta_{n}^{F, \tau}$ - since derived from the same sequence $\left\{X_{n}\right\}-$ are clearly not. Consider a fixed $t \in \mathbf{R}$. One implication of (1) is that $\alpha_{n}^{F}(t)$ and $\beta_{n}^{F, \tau}(t)$ are asymptotically independent. This may seem plausible, since they are deterministic transformations of $\mathbb{F}_{n}(t)$ and $\mathbb{F}_{n}(t / n)$, respectively, and it is known that the extreme and middle order statistics are asymptotically independent, cf. $[21]^{2)}$. But (1) states even stronger that the whole processes are asymptotically independent - and that although, for any fixed $n, \alpha_{n}^{F}$ and $\beta_{n}^{F, \tau}$ are linked via the strongest form of stochastic dependence: knowing one means knowing the other.

When it comes to proving the result, the first question arising is: weak convergence in which measurable space? Since we canonically take Borel $\sigma$-fields, it comes down to choosing a topological space, which desirably is metrizable and separable. The processes involved have discontinuous paths and the whole real line as their time domain. Thus, for example, the nice, separable metric space $\left(C[0,1],\|\cdot\|_{\infty}\right)$, the space of all continuous functions on $[0,1]$, is not an option. But the trajectories of all processes are right-continuous, and the left-hand limits exist in all points, i.e., they are càdlàg functions: «continue à droite, limites à gauche» (sometimes also rcll). The space of all càdlàg functions on the time domain $T$ is usually denoted by $D(T)$.

An element of $D(\mathbf{R})$ stays bounded on a compact set, just as a continuous function does. Hence the sup-metric $\|\cdot\|_{\infty}$ is a possible metric for $D[0,1]$. It induces the topology of uniform convergence or short, the uniform topology. However, this metric is unsuitable for $D[0,1]$, due to several reasons. First, $\left(D[0,1],\|\cdot\|_{\infty}\right)$ is not separable (see, e.g., [13, Chap. VI, § 1a, 1.2]). Second, and more severe, there are measurability problems. The empirical process is not measurable with respect to the uniform topology. In fact, Donsker's original proof of the weak convergence of the uniform empirical process was flawed, because he used this topology.

In 1956, Skorokhod [22] proposed several other topologies on $D[0,1]$, of which the $J_{1}$-topology has become the most popular. It is coarser than the uniform topology, separable, metrizable and solves the measurability issue. It allows for a workable Arzelà-Ascoli-type compactness characterization and it also declares a convergence more natural to functions with jumps. Nowadays, $D[0,1]$ is by default equipped with the $J_{1}$-topology and simply referred to as the Skorokhod space. We endow $D(\mathbf{R})$ with a proper extension of this $J_{1}$-topology (by the same means one declares a uniform

\footnotetext{
2) The authors thank E. Häusler for pointing out the reference.
} 
topology for functions on the real line, cf. p. 756) and then treat the convergence statement (1) in the Skorokhod product space $D(\mathbf{R}) \times D(\mathbf{R})$. The proof then breaks down into two tasks: Derive a weak convergence criterion in the space $D(\mathbf{R}) \times D(\mathbf{R})$ (Theorem 5.1) and show that $\left(\alpha_{n}^{F}, \beta_{n}^{F, \tau}\right)$ satisfies it (Section 6).

The standard method of proving weak convergence of stochastic processes is as follows: Prove the weak convergence of the finite-dimensional distributions, and show that the sequence is tight. The key argument here is Prokhorov's theorem [19]. For example, this method is used to show that the partial sum process converges to the Brownian motion in $\left(C[0,1],\|\cdot\|_{\infty}\right)$ (Donsker's theorem [6]). The principle transfers with little alteration to $D[0,1]$ and $D(\mathbf{R})$. We will show that it extends as well to $D(\mathbf{R}) \times D(\mathbf{R})$. It is, however, only feasible, if the finite-dimensional distributions are known, and there are other approaches as well, see, e.g., [13].

The paper is organized as follows. Section 2 states the task in detail, the principal statement of this paper is formulated in Theorem 2.1. The predominant rest of the paper is devoted to its proof: Section 3 introduces the space $D(\mathbf{R})$ and states the classic convergence criterion, Section 4 deals with tightness in $D(\mathbf{R})$. In Section 5 we begin to develop a short weak convergence theory for the product space $D(\mathbf{R}) \times D(\mathbf{R})$ and prove an analogous convergence criterion. Finally we apply the latter to show Theorem 2.1 in Section 6. The paper ends with Section 7 in which a short description is given how Theorem 2.1 can be used in statistics.

We conclude the introduction with some remarks on the literature. Most of what we use are classical results being covered in a variety of textbooks. Our main reference is Patrick Billingsley's «Convergence of Probability Measures» [3]. This book's first edition dates back to 1968 and features a stage-wise development from $C[0,1]$ via $D[0,1]$ to $D[0, \infty)$. A number of newer books, like, e.g., [18], [9], [30], and [13], consider right away the space $D[0, \infty)$, or a more general version of it, without paying extra attention to $D[0,1]$, and hence tend to be more profound. In particular, [13] gives an exhaustive treatment of weak convergence on $D[0, \infty)$.

Besides [22] the other two important papers on $D[0,1]$ are [16] and [19]. The analogue of $D[0, \infty)$ is due to Stone [24]. Billingsley [3] gives a construction of a complete metric on $D[0, \infty)$. He adopts a suggestion of Lindvall [17], who in turn follows Whitt's approach on $C[0, \infty)$, [28]. Whitt also suggests another metric on $D[0, \infty)$, [29].

2. Main result. Let $F$ be an arbitrary distribution function, and $X_{1}, X_{2}, \ldots$ a sequence of i.i.d. random variables being distributed according to $F$. The corresponding empirical distribution function is given by

$$
\mathbb{F}_{n}(t)=\frac{1}{n} \sum_{k=1}^{n} \mathbb{I}_{\left\{X_{k} \leqslant t\right\}}, \quad t \in \mathbf{R}, \quad n \geqslant 1
$$


The following family $\left\{\alpha_{n}^{F} \mid n \in \mathbf{N}\right\}$ of random functions is called the empirical process:

$$
\alpha_{n}^{F}(t)=\sqrt{n}\left(\mathbb{F}_{n}(t)-F(t)\right)=\frac{1}{\sqrt{n}} \sum_{k=1}^{n}\left(\mathbb{I}_{\left\{X_{k} \leqslant t\right\}}-F(t)\right), \quad t \in \mathbf{R}, \quad n \geqslant 1 .
$$

Furthermore, for any real number $\tau$, let

$$
\begin{aligned}
\beta_{n}^{F, \tau}(t)= \begin{cases}n\left[\mathbb{F}_{n}\left(\tau+\frac{t}{n}\right)-\mathbb{F}_{n}(\tau)\right], & \text { if } t \geqslant 0, \\
n\left[\mathbb{F}_{n}\left(\tau+\frac{t}{n}\right)-\mathbb{F}_{n}(\tau-)\right], & \text { if } t<0,\end{cases} \\
= \begin{cases}\sum_{k=1}^{n} \mathbb{I}_{\left(\tau, \tau+\frac{t}{n}\right]}\left(X_{k}\right), & \text { if } t \geqslant 0, \\
\sum_{k=1}^{n}-\mathbb{I}_{\left(\tau+\frac{t}{n}, \tau\right)}\left(X_{k}\right), & \text { if } t<0 .\end{cases}
\end{aligned}
$$

We want to call the family $\left\{\beta_{n}^{F, \tau} \mid n \in \mathbf{N}\right\}$ the rescaled empirical distribution function. Whenever it is clear or not of interest which $F$ and $\tau$ are meant, we will shortly write $\alpha_{n}$ and $\beta_{n}$. In Theorem 2.1 we will make the following basic assumptions on $F$.

Condition C.1. $F$ has both, left- and right-hand side, derivatives in $\tau$. Call the former $\varrho_{1}$ and the latter $\varrho_{2}$, i.e.,

$$
\begin{aligned}
& \varrho_{1}=\lim _{h \succ 0} \frac{F(\tau+h)-F(\tau-)}{h}, \\
& \varrho_{2}=\lim _{h \searrow 0} \frac{F(\tau+h)-F(\tau)}{h} .
\end{aligned}
$$

Pay attention to the $\tau-$ in (3). This definition of left-hand side derivative does not require $F$ to be continuous in $\tau$.

The next step is to specify the limit processes of $\left\{\alpha_{n}\right\}$ and $\left\{\beta_{n}\right\}$. Let $B_{0}=\left\{B_{0}(t) \mid t \in[0,1]\right\}$ be a Brownian bridge and

$$
B_{1}=B_{1}^{F}=B_{0} \circ F, \quad \text { i.e., } \quad B_{1}^{F}(t)=B_{0}(F(t)), \quad t \in \mathbf{R} .
$$

$B_{1}^{F}$ is a Gaussian process with expectation zero and covariance function $\operatorname{cov}(s, t)=F(s)(1-F(t))$ for $s \leqslant t$. Furthermore, let $N_{1}, N_{2}$ be two Poisson processes with the following properties:

(a) $N_{1}, N_{2}$, and $B_{1}$ are independent;

(b) $N_{i}$ has rate $\varrho_{i}, i=1,2$;

(c) $N_{2}$ has, as usual, right-continuous trajectories while those of $N_{1}$ are left-continuous, i.e., the value at a jump point is always set to the left-hand limit; note that this leaves the finite-dimensional distributions unchanged. 
Then define $N_{0}=N_{0}^{\varrho_{1}, \varrho_{2}}$ by

$$
N_{0}(t)= \begin{cases}-N_{1}(-t), & t<0 \\ N_{2}(t), & t \geqslant 0\end{cases}
$$

All four stochastic processes we have introduced so far, $\alpha_{n}, \beta_{n}, B_{1}$, and $N_{0}$, have trajectories in the Skorokhod space $D=D(\mathbf{R})$, that is, the space of all càdlàg functions on $\mathbf{R}$, equipped with the Skorokhod topology ( $J_{1}$-topology). The Skorokhod space is properly introduced in Section 3. Whenever we write $D$, the topological space is meant. This also applies to $D \times D$ (product topology). The Skorokhod space $D$ is a Polish space. Its Borel $\sigma$-field will be denoted by $\mathscr{D}$.

It is known and in the case of the uniform $(0,1)$ distribution considered to be folklore that

(A) $\alpha_{n}^{F} \stackrel{\mathscr{L}}{\longrightarrow} B_{1}^{F}$ in $D$, and

(B) if C.1 is satisfied, then $\beta_{n}^{F, \tau} \stackrel{\mathscr{L}}{\longrightarrow} N_{0}^{\varrho_{1}, \varrho_{2}}$ in $D$.

$\mathrm{R}$ e $\mathrm{m}$ a r k on (B). The definition (2) of $\left\{\beta_{n}\right\}$ resembles a sequence of difference quotients. It is therefore not surprising that the derivative of $F$ at $\tau$ appears as parameter in the limit process of $\left\{\beta_{n}\right\}$. But $F$ does not have to be differentiable at $\tau$, it may have a sharp bend at $\tau$ (i.e., left- and right-hand derivative differ) or even a jump (left- and right-hand limit differ). The behavior of the process $\beta_{n}$ on the positive half-axis is determined by the behavior of $F$ in the right-hand vicinity of $\tau$, likewise for the negative half-axis. Thus the assumption of differentiability can be weakened to Condition C.1.

In the proof of Proposition 6.2 it becomes clear that, instead of C.1, we only require the (formally) weaker condition that all of the limits

$\lim _{n \rightarrow \infty} \frac{n}{t}\left[F\left(\tau+\frac{t}{n}\right)-F(\tau-)\right]$ for $t<0, \quad \lim _{n \rightarrow \infty} \frac{n}{t}\left[F\left(\tau+\frac{t}{n}\right)-F(\tau)\right]$ for $t>0$

exist in order to have convergence of the process $\left\{\beta_{n}\right\}$. However, it can be shown that for monotone functions $F$ these limits coincide for all $t>0$ or all $t<0$, respectively, and, even more, that the left- and right-hand derivatives exist at $\tau$. Finally it should be noted that C.1 is a very weak condition for instance, it does not imply continuity in an upper or lower neighborhood of $\tau$.

The following result is new.

Theorem 2.1. Under Condition C.1 $\left(\alpha_{n}^{F}, \beta_{n}^{F, \tau}\right)$ converges in distribution to $\left(B_{1}^{F}, N_{0}^{\varrho_{1}, \varrho_{2}}\right)$ in $D \times D$.

$\mathrm{R}$ e $\mathrm{m}$ a r ks. (I) Keep in mind that we have defined $N_{0}$ to be independent of $B_{1}$, which specifies the distribution of $\left(B_{1}, N_{0}\right)$. The remarkable feature of Theorem 2.1 is not the convergence itself, but rather the fact, that 
the «highly dependent» $\alpha_{n}$ and $\beta_{n}$ (knowing one means knowing the other) converge to independent limits.

(II) Of course, (B) follows from Theorem 2.1. Note that - apart from the regularity condition C.1 $-F$ is completely arbitrary. The result does not seem to be contained as such in the literature. It should, however, be compared to Theorem 3.1 in [4]. The authors there consider processes of the type $n \mathbb{F}_{n}\left(a_{n} t+b_{n}\right)$, where $\left\{a_{n}\right\}$ and $\left\{b_{n}\right\}$ are sequences of real numbers such that the adjusted first order statistic $\left(X_{1: n}-b_{n}\right) / a_{n}$ converges to a nondegenerate limit. Such an extreme-value process may coincide with $\beta_{n}^{F, \tau}$ if $\tau$ is the left endpoint of the support of $F$ and $\left\{b_{n}\right\}$ is constant equal to $\tau$. In this situation, our Condition C.1 with $\varrho_{2}>0$ implies the assumption on $F$ in [4]: $F$ lies in the domain of attraction of the distribution function $L_{2,1}(x)=\left(1-e^{-x}\right) \mathbb{I}_{(0, \infty)}(x)$ (Weibull distribution with shape parameter 1), which is an extreme-value distribution of type 2 (cf., e.g., [12, section 2.4, Theorem 2.4.4]).

(III) Convergence in law is canonically defined on a Borel $\sigma$-field, the underlying topological space being here $D \times D$. On the other hand, $\left(\alpha_{n}, \beta_{n}\right)$ and $\left(B_{1}, N_{0}\right)$ are pairs of random variables and hence defined on the product measure space, i.e., the $\sigma$-field $\mathscr{D} \otimes \mathscr{D}$. Fortunately, $\mathscr{D} \otimes \mathscr{D}$ coincides with the Borel $\sigma$-field on $D \times D$ (cf. Lemma 5.1).

The proof of Theorem 2.1 is subject of Section 6 .

3. Weak convergence in $D$. Preliminary note: Most textbooks and articles consider the space $D[0, \infty)$ instead of $D(\mathbf{R})$. Both spaces are qualitatively equal, all results for $D[0, \infty)$ hold with little notational change (which demands its due amount of care) also for $D(\mathbf{R})$. Define

$$
D=D(\mathbf{R})=\{x: \mathbf{R} \rightarrow \mathbf{R} \mid x(t-), x(t+) \text { exist, } x(t)=x(t+) \forall t \in \mathbf{R}\} .
$$

Elements of $D$ have at most countably many discontinuity points and are bounded on compact sets. We declare a topology on $D$ by the following characterization of convergence. Let $\Lambda$ denote the class of all strictly increasing, continuous, surjective mappings $\lambda$ from $\mathbf{R}$ onto itself. A sequence $\left\{x_{n}\right\} \subset D$ converges to $x \in D$ if and only if a sequence $\left\{\lambda_{n}\right\} \subset \Lambda$ exists such that

$$
\begin{cases}\lambda_{n}(t) \rightarrow t & \text { uniformly in } t \in \mathbf{R}, \\ x_{n}\left(\lambda_{n}(t)\right) \rightarrow x(t) & \text { uniformly in } t \in[-m, m] \text { for all } m \in \mathbf{N} .\end{cases}
$$

This is a $D(\mathbf{R})$-version of the $J_{1}$-topology, originated by Skorokhod [22]. This is the only topology we consider on $D$ and subsequently refer to it as the Skorokhod topology. For details on different topologies on $D$ see, for example, [20]. Compare the above characterization to [13, Chap. VI, $\S 1$ b, Theorem 1.14]. Note that, unlike in $D[0, \infty)$, the point 0 must not play a special role in $D(\mathbf{R})$. 
Since the identity is an element of $\Lambda$, uniform convergence on compact sets implies Skorokhod convergence. In fact, the Skorokhod topology is strictly coarser than the topology of locally uniform convergence (uniform topology), i.e., there are fewer open sets and more convergent sequences. For instance, $\left\{\mathbb{1}_{[1 / n, \infty)} \mid n \in \mathbf{N}\right\}$ converges in the Skorokhod topology, but not in the uniform topology. As mentioned before, by writing $D$ we always mean the topological space. Let $\mathscr{D}$ be its Borel $\sigma$-field. The topological space $D$ is separable (whereas the set $D$ endowed with the uniform topology is not separable), completely metrizable and in this sense a Polish space. See, e.g., [3] for a complete metric.

Let $\pi_{t}$ denote the projection $\pi_{t}: D \rightarrow \mathbf{R}: x \mapsto x(t)$. For any probability measure $P$ on $(D, \mathscr{D})$ let $T_{P}$ be the set of all points $t \in \mathbf{R}$ for which $\pi_{t}$ is $P$-almost surely continuous. All random variables we consider are defined on a common probability space with probability measure $\mathbf{P}$. With $\mathbf{P} \circ X^{-1}$ being the distribution of a random variables $X$ in $D$ we write $T_{X}$ for $T_{\mathbf{P} \circ X^{-1}}$.

A version of the following lemma can be found in [3, Section 16].

Lemma 3.1. The complement of $T_{X}$ in $\mathbf{R}$ is at most countable.

It follows that $T_{X}$ is dense.

$\mathrm{D}$ e $\mathrm{f}$ i $\mathrm{n}$ i t i o $\mathrm{n}$ 3.1. In $D$ we say that the finite-dimensional distributions of $X_{n}$ converge to those of $X$, and write $X_{n} \stackrel{\mathscr{F}}{\longrightarrow} X$, if

$$
\left(X_{n}\left(t_{1}\right), \ldots, X_{n}\left(t_{k}\right)\right) \stackrel{\mathscr{L}}{\longrightarrow}\left(X\left(t_{1}\right), \ldots, X\left(t_{k}\right)\right)
$$

for all $k \in \mathbf{N}$ and $t_{1}<\cdots<t_{k} \in T_{X}$.

R e m a r ks. (I) We restrict $t_{1}, \ldots, t_{k}$ to lie in $T_{X}$, because $X_{n} \stackrel{\mathscr{L}}{\longrightarrow} X$ in $D$ does not necessarily imply $\pi_{t}\left(X_{n}\right) \stackrel{\mathscr{L}}{\longrightarrow} \pi_{t}(X)$, cf., e.g., [13, Chap. VI, $\S 3 \mathrm{~b}$, Proposition 3.14]. This is due to the fact that $\pi_{t}$ (function from $D$ to $\mathbf{R}$ ) is continuous at a point $x$ only if $x$ (function from $\mathbf{R}$ to $\mathbf{R}$ ) is continuous at $t$, cf. [3, p. 134, Theorem 12.5(i)]. Think, for instance, of $\mathbb{I}_{[1 / n, \infty)} \rightarrow \mathbb{I}_{[0, \infty)}$, but $\pi_{0}\left(\mathbb{I}_{[1 / n, \infty)}\right) \nrightarrow \pi_{0}\left(\mathbb{I}_{[0, \infty)}\right)$.

(II) Definition 3.1 is equivalent to: there exists a dense subset $S$ of $\mathbf{R}$ such that (6) holds for all finite subsets $\left\{t_{1}, \ldots, t_{k}\right\}$ of $S$, see [13, Chap. VI, $\S 3$ b, Lemma 3.19]. In this sense $\stackrel{\mathscr{F}}{\longrightarrow}$ does not depend on its right-hand side.

Now here is a characterization of weak convergence in $D$. It is phrased in terms of random variables and convergence in law — which is equivalent to the weak convergence of the respective distributions.

Proposition 3.1. Let $\left\{X_{n}\right\}$ be a sequence of random variables in $(D, \mathscr{D})$ with the following two properties:

1) $\left\{X_{n}\right\}$ is tight;

2) $X_{n} \stackrel{\mathscr{F}}{\longrightarrow} X$.

Then $X_{n} \stackrel{\mathscr{L}}{\longrightarrow} X$. 
Short, convergence of the finite-dimensional distributions (in the sense of Definition 3.1) and tightness together imply convergence in law. These two conditions are sufficient and necessary, cf., e.g., [13, Chap. VI, §3b, 3.20] or $[3$, p. 139].

4. Tightness in $D$. In order to make use of Proposition 3.1 we need a handy tightness criterion. Recall tightness of a sequence: A family $\mathscr{P}$ of probability measures on the Borel $\sigma$-field of a metric space is tight, if for every $\varepsilon>0$ there exists a compact set $K$ such that $P(K)>1-\varepsilon$ for every $P \in \mathscr{P}$. A family of random variables is tight if the family of their respective distributions is tight. Prokhorov's theorem tells us that in complete metric spaces, like $D$, tightness is equivalent to relative compactness. A family $\mathscr{P}$ of probability measures is relatively compact, if every sequence in $\mathscr{P}$ contains a convergent subsequence. The limit needs not to lie in $\mathscr{P}$.

We present three criteria which allow one to confirm that a given sequence of random variables in $D$ is tight. The first is, in fact, a characterization of tightness.

4.1. A tightness characterization in $D$. First we need to introduce some notation. We will deal with intervals of the type $[-m, m]$, where $m \in \mathbf{N}$.

For an arbitrary function $x: \mathbf{R} \rightarrow \mathbf{R}$ and an arbitrary set $T \subset \mathbf{R}$ we define

$$
w(x, T)=\sup _{s, t \in T}|x(s)-x(t)| .
$$

We call any finite set $\sigma=\left\{s_{0}, \ldots, s_{k}\right\} \subset \mathbf{R}$ satisfying $-m=s_{0}<s_{1}<\cdots<$ $s_{k}=m$ a grid on $[-m, m]$. If

$$
s_{i}-s_{i-1}>\delta \quad \text { for all } i=2, \ldots, k-1,
$$

i.e., all intervals except those at the left and right end are wider than $\delta$, then we call the grid $\delta$-sparse. Let $\mathscr{S}(m, \delta)$ be the set of all $\delta$-sparse grids on $[-m, m]$ and define the following modulus:

$$
\widehat{w}_{m}(x, \delta)=\inf _{\mathscr{S}(m, \delta)} \max _{1 \leqslant i \leqslant k} w\left(x,\left[s_{i-1}, s_{i}\right)\right) .
$$

Theorem 4.1. A sequence of random variables $\left\{X_{n}\right\}$ in $(D, \mathscr{D})$ is tight if and only if the following two conditions hold:

1) For all $t$ in a dense subset $T_{0}$ of $\mathbf{R}$,

$$
\lim _{a \rightarrow \infty} \limsup _{n} \mathbf{P}\left(\left|X_{n}(t)\right| \geqslant a\right)=0,
$$

2) for every $m \in \mathbf{N}$ and $\varepsilon>0$,

$$
\lim _{\delta \rightarrow 0} \limsup _{n} \mathbf{P}\left(\widehat{w}_{m}\left(X_{n}, \delta\right) \geqslant \varepsilon\right)=0 .
$$

P r o o f. Cf. [3, Theorem 16.8] in combination with the subsequent corollary. 


\subsection{A moment-type tightness criterion.}

Proposition 4.1. Let $X$ and $X_{n}, n \in \mathbf{N}$, be random variables in $(D, \mathscr{D})$. Suppose that

1) $X_{n} \stackrel{\mathscr{F}}{\longrightarrow} X$, and

2) there exist a nondecreasing continuous function $H: \mathbf{R} \rightarrow \mathbf{R}$ and real numbers $a>1$ and $b \geqslant 0$ such that

$$
\mathbf{E}\left(\left|X_{n}(s)-X_{n}(r)\right|^{b}\left|X_{n}(t)-X_{n}(s)\right|^{b}\right) \leqslant(H(t)-H(r))^{a}
$$

holds for all $r<s<t$ and $n \geqslant 1$.

Then $\left\{X_{n}\right\}$ is tight.

P r o o f. The $D[0,1]$ version of this proposition is Theorem 13.5 on p. 142 in [3]. The proof is also worked out in detail for the $D(\mathbf{R})$-case in $[27]$.

It needs to be shown that Proposition 4.1, 1), 2) implies Theorem 4.1, 1), 2). In fact, Theorem 4.1, 1) follows from Proposition 4.1, 1) already, and Theorem 4.1,2) follows from Proposition 4.1,2). And of course, by Proposition 3.1, under the assumptions of Proposition 4.1 we have $X_{n} \stackrel{\mathscr{L}}{\longrightarrow} X$ in $(D, \mathscr{D})$.

4.3. A point-process tightness criterion. Let $\mathscr{T}$ be the set of all nondecreasing series $\left\{t_{z} \mid z \in \mathbf{Z}\right\}$ that meet the restrictions $t_{z} \in[-\infty, \infty]$ for all $z \in \mathbf{Z}, t_{0} \leqslant 0<t_{1}, t_{z} \rightarrow \pm \infty$ as $z \rightarrow \pm \infty$, and $\left\{t_{z}\right\}$ is strictly increasing where it is not $\pm \infty$. Then define the following two classes of functions:

$$
\begin{aligned}
\mathscr{V}^{+1}= & \left\{x: \mathbf{R} \rightarrow \mathbf{R} \mid x=c-\sum_{z=-\infty}^{0} \mathbb{I}_{\left(-\infty, t_{z}\right)}+\sum_{z=1}^{\infty} \mathbb{I}_{\left(t_{z}, \infty\right)}, c \in \mathbf{Z},\left\{t_{z}\right\} \in \mathscr{T}\right\}, \\
\mathscr{V}^{+}= & \left\{x: \mathbf{R} \rightarrow \mathbf{R} \mid x=c-\sum_{z=-\infty}^{0} c_{z} \mathbb{I}_{\left(-\infty, t_{z}\right)}+\sum_{z=1}^{\infty} c_{z} \mathbb{I}_{\left(t_{z}, \infty\right)},\right. \\
& \left.c \in \mathbf{Z},\left\{t_{z}\right\} \in \mathscr{T}, c_{z} \in \mathbf{N} \text { for all } z \in \mathbf{Z}\right\} .
\end{aligned}
$$

Apparently $\mathscr{V}^{+1} \subset \mathscr{V}^{+} \subset D$. The set $\mathscr{V}^{+}$allows also the following characterization: it contains all elements of $D$ that are nondecreasing and integer-valued. Then, by employing (5), it is easy to see that the potential limit of any series $\left\{x_{n}\right\}$ in $\mathscr{V}^{+}$has this property, too. Hence $\mathscr{V}^{+}$is closed in $D$ and therefore measurable. As for $\mathscr{V}^{+1}$, note that Skorokhod convergence $x_{n} \rightarrow x$ implies that for all $t \in \mathbf{R}$ there exists a sequence $\left\{t_{n}\right\} \subset \mathbf{R}$ such that $t_{n} \rightarrow t$ and

$$
x_{n}\left(t_{n}\right)-x_{n}\left(t_{n}-\right) \longrightarrow x(t)-x(t-),
$$

cf. [13, Chap. VI, $\S 2 \mathrm{a}$, Proposition 2.1]. Hence, if $\left\{x_{n}\right\} \subset \mathscr{V}^{+1}$, then the limit $x$ can only have jumps of size 1 as well: $\mathscr{V}^{+1}$ is closed in $D$. We call a random function whose paths lie almost surely in $\mathscr{V}^{+1}$ a counting process. 
Proposition 4.2. Let $X$ and $X_{n}, n \in \mathbf{N}$, be random variables in $(D, \mathscr{D})$. Suppose that

1) $X_{n} \stackrel{\mathscr{F}}{\longrightarrow} X$

2) $\mathbf{P}\left(X \in \mathscr{V}^{+1}\right)=1$, and

3) $\mathbf{P}\left(X_{n} \in \mathscr{V}^{+}\right)=1, n \in \mathbf{N}$.

Then $\left\{X_{n}\right\}$ is tight.

This is generalization of Theorem 3.37 in [13, Chap. VI, $\S 3 \mathrm{~b}]$. Basically, [13] consider the space $D[0, \infty)$ and require $X_{n}, n \in \mathbf{N}$, also to be counting processes in the above sense. A proposition of exactly the same type as ours ( $X$ has jumps of size $1, X_{n}$ has integer-valued jumps) can be found in [4]. For the sake of completeness we present an alternative proof.

Proof of Proposition 4.2. We apply Theorem 4.1. The implication Proposition $4.2,1) \Longrightarrow$ Theorem $4.1,1$ ) is straightforward. It remains to derive condition 2) of Theorem 4.1.

Let $m \in \mathbf{N}$ and initially also $\delta>0$ be fixed. Then choose a $\delta$-sparse grid $\sigma=\left\{s_{0}, \ldots, s_{k}\right\}$ on $[-m, m]$ according to the following additional restrictions:

$$
s_{i}-s_{i-1}<2 \delta, \quad i=1, \ldots, k, \quad \text { and } \quad s_{1}, \ldots, s_{k-1} \in T_{X} .
$$

The latter is always possible, since $T_{X}$ is dense in $\mathbf{R}$, but $s_{0}=-m \in T_{X}$ or $s_{k}=m \in T_{X}$ does not need to hold. Now define the following two sets:

$$
\begin{aligned}
A & =\left\{\left(t_{1}, \ldots, t_{k-1}\right) \mid t_{i+1}-t_{i-1}<\frac{3}{2}, i=2, \ldots, k-2\right\} \subset \mathbf{R}^{k-1}, \\
\widetilde{A}_{\sigma} & =\left\{x \in \mathscr{V}^{+} \mid\left(x\left(s_{1}\right), \ldots, x\left(s_{k-1}\right)\right) \in A\right\} \subset \mathscr{V}^{+} .
\end{aligned}
$$

With these constructions the proof breaks down into two steps. First we show

(a) $\lim \sup _{n} \mathbf{P}\left(\widehat{w}_{m}\left(X_{n}, \delta\right) \geqslant \varepsilon\right) \leqslant \mathbf{P}\left(X \notin \widetilde{A}_{\sigma}\right)$ for all positive $\varepsilon$ and then

(b) $\mathbf{P}\left(X \notin \widetilde{A}_{\sigma}\right) \rightarrow 0$ as $\delta \rightarrow 0$. implies

Part (a). By construction of $\widetilde{A}_{\sigma}$ we have $x \in \widetilde{A}_{\sigma} \Rightarrow \widehat{w}_{m}(x, \delta)=0$, which

$$
\mathbf{P}\left(X_{n} \notin \widetilde{A}_{\sigma}\right) \geqslant \mathbf{P}\left(\widehat{w}_{m}\left(X_{n}, \delta\right) \geqslant \varepsilon\right) \quad \forall n \in \mathbf{N}, \varepsilon>0 .
$$

Since $s_{i}, i=1, \ldots, k-1$, have been chosen in $T_{X}$, we have

$$
\left(X_{n}\left(s_{1}\right), \ldots, X_{n}\left(s_{k-1}\right)\right) \stackrel{\mathscr{L}}{\longrightarrow}\left(X\left(s_{1}\right), \ldots, X\left(s_{k-1}\right)\right) .
$$

The set $A$ is open in $\mathbf{R}^{k-1}$, and hence by the portmanteau theorem

$$
\mathbf{P}\left(X \notin \widetilde{A}_{\sigma}\right) \geqslant \limsup \mathbf{P}\left(X_{n} \notin \widetilde{A}_{\sigma}\right) \geqslant \limsup \mathbf{P}\left(\widehat{w}_{m}\left(X_{n}, \delta\right) \geqslant \varepsilon\right) \quad \forall \varepsilon>0 .
$$


Part (b). Define $T_{z}, z \in \mathbf{Z}$, to be the jump times of $X$ (we understand them as random variables in $[-\infty, \infty])$, where we count as follows:

$$
\cdots<T_{-2}<T_{-1}<T_{0} \leqslant 0<T_{1}<T_{2}<\cdots
$$

Now consider the following events:

$$
\begin{aligned}
B_{n} & =\left\{T_{-n}<-m, m<T_{n}\right\}, \quad n \in \mathbf{N}, \\
C_{\delta, n} & =\left\{T_{i}-T_{i-1}>4 \delta, i=-n+1, \ldots, 0, \ldots, n\right\}, \quad n \in \mathbf{N}, \quad \delta>0 .
\end{aligned}
$$

It holds (and is proved below) that

$$
\begin{aligned}
& \forall \varepsilon>0 \exists n \in \mathbf{N}: \mathbf{P}\left(B_{n}\right) \geqslant 1-\frac{\varepsilon}{2}, \\
& \forall \varepsilon>0, n \in \mathbf{N} \exists \delta>0: \mathbf{P}\left(C_{\delta, n}\right) \geqslant 1-\frac{\varepsilon}{2},
\end{aligned}
$$

and

$$
\left\{X \in \mathscr{V}^{+1}\right\} \cap B_{n} \cap C_{\delta, n} \subset\left\{X \in \widetilde{A}_{\sigma}\right\} .
$$

Since $\mathbf{P}\left(X \in \mathscr{V}^{+1}\right)=1,(9),(10)$ and (11) imply together

$$
\forall \varepsilon>0 \exists \delta>0: \mathbf{P}\left(X \notin \widetilde{A}_{\sigma}\right) \leqslant \varepsilon .
$$

It remains to show (9) and (10). Both follow by the same principle from the fact that $X$ is a counting process, which we will exemplify at (9). Assume the opposite is true: $\exists \varepsilon>0 \forall n \in \mathbf{N}: \mathbf{P}\left(B_{n}\right)<1-\varepsilon / 2$. Since $\left\{B_{n} \mid n \in \mathbf{N}\right\}$ is an increasing series of sets, $\mathbf{P}\left(\bigcap_{n} \mathrm{C} B_{n}\right) \geqslant \varepsilon / 2$, where $C$ means set complement. The event $\bigcap_{n} \mathrm{C} B_{n}$ reads as: all $T_{z}, z \in \mathbf{Z}$, lie in $[-m, m]$. By definition of the set $\mathscr{T}$ this is a contradiction to $X \in \mathscr{V}^{+1}$. Proposition 4.2 is proved.

5. Weak convergence in $D \times D$. In Theorem 5.1 we will give a weak convergence characterization in $D \times D$ of the same type as Proposition 3.1. The set $D \times D$ is the collection of all pairs $(x, y)$, where $x, y \in D$. It is endowed with the product topology, i.e.,

$$
\left(x_{n}, y_{n}\right) \rightarrow(x, y) \Longleftrightarrow\left\{\begin{array}{l}
x_{n} \rightarrow x, \\
y_{n} \rightarrow y .
\end{array}\right.
$$

Again, by writing $D \times D$ we refer to the topological space. $D \times D$ is a Polish space. The following is important.

Lemma 5.1. The Borel $\sigma$-field on $D \times D$ coincides with the product $\sigma$-field $\mathscr{D} \otimes \mathscr{D}$.

P r o o f. See, e.g., [10, Theorem 5.10, p. 115]. Separability is needed.

$\mathrm{R}$ e $\mathrm{m}$ a $\mathrm{r} \mathrm{k}$. One can identify the pair of functions $(x, y)$ with the function $f_{x, y}: \mathbf{R} \rightarrow \mathbf{R}^{2}: t \mapsto(x(t), y(t))$. Note that $x, y \in D$ if and only if $f_{x, y}$ is a càdlàg function from $\mathbf{R}$ to $\mathbf{R}^{2}$. We call the space of such functions 
$D\left(\mathbf{R}, \mathbf{R}^{2}\right)$. The generalization is straightforward: The convergence characterization reads exactly as (5), only $x_{n}\left(\lambda_{n}(t)\right)$ and $x(t)$ are $\mathbf{R}^{2}$-valued. In fact the co-domain can easily be replaced by any Polish space without having to change anything.

If we identify $(x, y) \leftrightarrow f_{x, y}$, then the sets $D \times D$ and $D\left(\mathbf{R}, \mathbf{R}^{2}\right)$ are equal, but the Skorokhod topology on $D\left(\mathbf{R}, \mathbf{R}^{2}\right)$ is strictly finer than the product topology on $D \times D$, i.e. it has less convergent sequences. Take, for instance, $x_{n}=\mathbb{I}_{[1 / n, \infty)}, y_{n}=\mathbb{1}_{[-1 / n, \infty)}$. However, both topologies induce the same Borel $\sigma$-field, cf. [20]. In this paper we are not at all concerned with the space $D\left(\mathbf{R}, \mathbf{R}^{2}\right)$. We deal with pairs of random variables and their convergence in law, which we want to be of the same type as (12). The product topology has to be our concern.

Theorem 5.1. Let $\left\{Z_{n}=\left(X_{n}, Y_{n}\right)\right\}$ be a sequence of random variables in $(D \times D, \mathscr{D} \otimes \mathscr{D})$. If

1) the sequences $\left\{X_{n}\right\}$ and $\left\{Y_{n}\right\}$ are tight, and

2) there is a random variable $Z=(X, Y)$ in $(D \times D, \mathscr{D} \otimes \mathscr{D})$ such that

$$
\begin{aligned}
& \left(X_{n}\left(t_{1}\right), \ldots, X_{n}\left(t_{k}\right), Y_{n}\left(t_{1}\right), \ldots, Y_{n}\left(t_{k}\right)\right) \\
& \quad \stackrel{\mathscr{L}}{\longrightarrow}\left(X\left(t_{1}\right), \ldots, X\left(t_{k}\right), Y\left(t_{1}\right), \ldots, Y\left(t_{k}\right)\right) \\
& \text { for all } k \in \mathbf{N}, t_{1}, \ldots, t_{k} \in T_{X} \cap T_{Y},
\end{aligned}
$$

then $Z_{n} \stackrel{\mathscr{L}}{\longrightarrow} Z$.

The rest of the section is devoted to the proof of Theorem 5.1. It is more convenient to formulate the proof in terms of probability measures than random variables. Therefore, let $P, P_{n}, P^{(1)}, P_{n}^{(1)}, P^{(2)}$, and $P_{n}^{(2)}$ be the distributions of $Z, Z_{n}, X, X_{n}, Y$, and $Y_{n}, n \in \mathbf{N}$, respectively.

One thing to note about the theorem is that in Theorem 5.1,1) we only require $\left\{X_{n}\right\}$ and $\left\{Y_{n}\right\}$ individually to be tight. This of course implies tightness of the joint sequence.

Lemma 5.2. If $\left\{X_{n}\right\}$ and $\left\{Y_{n}\right\}$ are tight sequences of random variables in $(D, \mathscr{D})$, then $\left\{Z_{n}=\left(X_{n}, Y_{n}\right)\right\}$ is tight in $(D \times D, \mathscr{D} \otimes \mathscr{D})$.

P r o o f. The lemma is a corollary of Tikhonov's theorem. Indeed, for any $\varepsilon>0$ we find compact sets $K_{1}, K_{2} \subset D$ such that $P_{n}^{(i)}\left(K_{i}\right)>1-\varepsilon / 2$ for all $n \in \mathbf{N}, i=1,2$, then $P_{n}\left(K_{1} \times K_{2}\right)>1-\varepsilon$ for all $n \in \mathbf{N}$, and $K_{1} \times K_{2}$ is compact in $D \times D$ by Tikhonov's theorem.

We now introduce projections. Let $T=\left\{t_{1}, \ldots, t_{k}\right\}$ and $S=$ $\left\{s_{1}, \ldots, s_{l}\right\}$, where $t_{1}<\cdots<t_{k}$ and $s_{1}<\cdots<s_{l}$. Define

$$
\begin{aligned}
& \pi_{T}: D \longrightarrow \mathbf{R}^{k}: x \mapsto\left(x\left(t_{1}\right), \ldots, x\left(t_{k}\right)\right)=\left(\pi_{t_{1}}(x), \ldots, \pi_{t_{k}}(x)\right), \\
& \begin{aligned}
\pi_{S, T}: D \times D \rightarrow \mathbf{R}^{l+k}:(x, y) & \mapsto\left(\pi_{S}(x), \pi_{T}(y)\right) \\
& =\left(x\left(s_{1}\right), \ldots, x\left(s_{l}\right), y\left(t_{1}\right), \ldots, y\left(t_{k}\right)\right) .
\end{aligned}
\end{aligned}
$$


Then Theorem 5.1, 2) can be written as

$$
P_{n} \circ \pi_{T, T}^{-1} \stackrel{\mathscr{L}}{\longrightarrow} P \circ \pi_{T, T}^{-1} \quad \forall T \subset T_{X} \cap T_{Y}, \quad|T|<\infty .
$$

Lemma 5.3. If $T \subset T_{X} \cap T_{Y}$, $T$ finite, then $\pi_{T, T}$ is P-a.e. continuous.

$\mathrm{P}$ r o o f. Let $A$ be the discontinuity set of $\pi_{T}$, i.e., the set of all points $x \in D$ in which $\pi_{T}$ is not continuous. The function $\pi_{T, T}$ is continuous at a point $(x, y) \in D \times D$ if and only if $\pi_{T}$ is continuous at $x$ and $y$. Hence the discontinuity set of $\pi_{T, T}$ is $(A \times D) \cup(D \times A)$. Due to our assumption $T \subset T_{X} \cap T_{Y}$ we have

$$
P((A \times D) \cup(D \times A)) \leqslant P(A \times D)+P(D \times A)=P^{(1)}(A)+P^{(2)}(A)=0 .
$$

The proof of Theorem 5.1 requires furthermore a few measuretheoretical concepts.

$\mathrm{D}$ e f i n i t i o n 5.1. Let $(\Omega, \mathscr{A})$ be a measurable space. Any subclass $\mathscr{S}$ of $\mathscr{A}$ that satisfies

$$
\left.\mu\right|_{\mathscr{S}}=\left.\nu\right|_{\mathscr{S}} \Rightarrow \mu=\nu
$$

for any two probability measures $\mu$ and $\nu$ on $\mathscr{A}$ is called a separating class for $\mathscr{A}$.

If $\mu$ and $\nu$ differ, then $\mathscr{S}$ already suffices to separate them. Recall that if a system of sets $\mathscr{S} \subset \mathscr{A}$ generates the $\sigma$-field $\mathscr{A}$ and is closed under the formation of finite intersections (i.e., is a $\pi$-system), then $\mathscr{S}$ is a separating class for $\mathscr{A}$, cf., e.g., [3, p. 9].

Lemma 5.4. If $\mathscr{S}_{1}$ and $\mathscr{S}_{2}$ are separating classes for the $\sigma$-fields $\mathscr{A}_{1}$ and $\mathscr{A}_{2}$, respectively, then so is $\mathscr{S}_{1} \times \mathscr{S}_{2}$ for $\mathscr{A}_{1} \otimes \mathscr{A}_{2}$.

$\mathrm{P}$ r o o f. We have to show that the two properties, $\pi$-system and generating class, extend from the marginals to the product. The former is apparent, for the latter see, e.g., [2, Theorem 22.1, p. 151].

For any $T_{0} \subset \mathbf{R}$ let

$$
\begin{aligned}
& \mathscr{F}\left(T_{0}\right)=\left\{\pi_{T}^{-1}(A)\left|A \in \mathscr{B}\left(\mathbf{R}^{|T|}\right), T \subset T_{0},\right| T \mid<\infty\right\}, \\
& \mathscr{H}\left(T_{0}\right)=\left\{\pi_{T, T}^{-1}(A)\left|A \in \mathscr{B}\left(\mathbf{R}^{2|T|}\right), T \subset T_{0},\right| T \mid<\infty\right\} .
\end{aligned}
$$

$\mathscr{F}\left(T_{0}\right)$ and $\mathscr{H}\left(T_{0}\right)$ are subclasses of $\mathscr{D}$ and $\mathscr{D} \otimes \mathscr{D}$, respectively, cf. [3, Theorem 16.6]. Roughly, the next two lemmas tell that $\mathscr{F}\left(T_{0}\right)$ and $\mathscr{H}\left(T_{0}\right)$ are «large enough», if $T_{0}$ is «large enough».

Lemma 5.5. If $T_{0}$ is dense in $\mathbf{R}$, then $\mathscr{F}\left(T_{0}\right)$ is a separating class for $\mathscr{D}$.

P r o o f. See [3, p. 170, Theorem 16.6].

Lemma 5.6. If $T_{0}$ is dense in $\mathbf{R}$, then $\mathscr{H}\left(T_{0}\right)$ is a separating class for $\mathscr{D} \otimes \mathscr{D}$. 
$\mathrm{P}$ r o o f. By Lemmas 5.4 and $5.5, \mathscr{F}\left(T_{0}\right) \times \mathscr{F}\left(T_{0}\right)$ is a separating class for $\mathscr{D} \otimes \mathscr{D}$. It remains to see that $\mathscr{F}\left(T_{0}\right) \times \mathscr{F}\left(T_{0}\right) \subset \mathscr{H}\left(T_{0}\right)$. To this end we introduce the class

$$
\mathscr{G}\left(T_{0}\right)=\left\{\pi_{S, T}^{-1}(A)\left|A \in \mathscr{B}\left(\mathbf{R}^{|S|+|T|}\right), S, T \subset T_{0},\right| S|,| T \mid<\infty\right\} .
$$

Evidently $\mathscr{F}\left(T_{0}\right) \times \mathscr{F}\left(T_{0}\right) \subset \mathscr{G}\left(T_{0}\right)$. Furthermore it holds $\mathscr{G}\left(T_{0}\right)=\mathscr{H}\left(T_{0}\right)$. This is because any set $\pi_{S, T}^{-1}(A) \in \mathscr{G}\left(T_{0}\right)$ can also be written as $\pi_{T \cup S, T \cup S}^{-1}(C)$ for an appropriate set $C \subset \mathbf{R}^{2|T \cup S|}$.

This concludes the preliminaries, and we present the proof of Theorem 5.1.

P r o of of $\mathrm{Th}$ e or e m 5.1. We have, $\left\{P_{n}^{(1)}\right\}$ and $\left\{P_{n}^{(2)}\right\}$ are both tight, hence $\left\{P_{n}=\left(P_{n}^{(1)}, P_{n}^{(2)}\right)\right\}$ is tight (Lemma 5.2). By Prokhorov's theorem, $\left\{P_{n}\right\}$ is relatively compact. (Here it is important that $\mathscr{D} \otimes \mathscr{D}$ coincides with the Borel $\sigma$-field on $D \times D$, cf. Lemma 5.1.) To each subsequence $\left\{P_{n^{\prime}}\right\}$ there exists a further (sub-)subsequence $\left\{P_{n^{\prime \prime}}\right\}$ that converges, i.e., there is a probability measure $Q=\left(Q^{(1)}, Q^{(2)}\right)$ on $(D \times D, \mathscr{D} \otimes \mathscr{D})$ (which of course depends on $\left.\left\{n^{\prime \prime}\right\}\right)$ such that

$$
P_{n^{\prime \prime}} \stackrel{\mathscr{L}}{\longrightarrow} Q
$$

Lemma 5.3 allows us to apply the continuous mapping theorem:

$$
P_{n^{\prime \prime}} \circ \pi_{T, T}^{-1} \stackrel{\mathscr{L}}{\longrightarrow} Q \circ \pi_{T, T}^{-1} \quad \text { for all finite } T \subset T_{Q^{(1)}} \cap T_{Q^{(2)}} .
$$

On the other hand, condition 2) of the Theorem 5.1 implies

$$
P_{n^{\prime \prime}} \circ \pi_{T, T}^{-1} \stackrel{\mathscr{L}}{\longrightarrow} P \circ \pi_{T, T}^{-1} \quad \text { for all finite } T \subset T \subset T_{P^{(1)}} \cap T_{P^{(2)}} .
$$

This means that if we let $T_{0}=T_{Q^{(1)}} \cap T_{Q^{(2)}} \cap T_{P^{(1)}} \cap T_{P^{(2)}}$, then $P$ and $Q$ agree on $\mathscr{H}\left(T_{0}\right)$. The set $T_{0}$ is dense in $\mathbf{R}$ (corollary of Lemma 3.1), thus $\mathscr{H}\left(T_{0}\right)$ is a separating class for $\mathscr{D} \otimes \mathscr{D}$ (Lemma 5.6), hence $P=Q$.

Thus we know that all subsequences of $\left\{P_{n}\right\}$ contain a weakly convergent sub-subsequence, and all of these sub-subsequences converge to the same limit $P$. It follows that $P_{n}$ converges weakly to $P$, cf. [3, Theorem 2.6, p. 20].

6. Proof of Theorem 2.1. By applying Theorem 5.1 the proof comes down to showing

(A) $\left\{\alpha_{n}^{F}\right\}$ is tight,

(B) $\left\{\beta_{n}^{F, \tau}\right\}$ is tight, and

(C) the finite-dimensional distributions of $\left(\alpha_{n}^{F}, \beta_{n}^{F, \tau}\right)$ converge to those of $\left(B_{1}^{F}, N_{0}^{\varrho_{1}, \varrho_{2}}\right)$ in the sense of Theorem 5.1,2).

Part (B) is an immediate corollary of point-process tightness criterion 4.2. The result (A) is not equally straightforward: In a first step we 
use the moment-type criterion 4.1 to show that it holds for continuous $F$ (Lemma 6.1), and then, building on that, show it for arbitrary distribution functions $F$ (Proposition 6.1). Part (C) is subject of Proposition 6.2.

Lemma 6.1. If $F$ is continuous, then the series of random variables $\left\{\alpha_{n}^{F}\right\}$ in $(D, \mathscr{D})$ is tight.

P r o o f. Some straightforward calculations yield

$$
\mathbf{E}\left(\left|\alpha_{n}(s)-\alpha_{n}(r)\right|^{2}\left|\alpha_{n}(t)-\alpha_{n}(s)\right|^{2}\right) \leqslant 6(F(t)-F(r))^{2}
$$

for all $r<s<t$ and all $n \in \mathbf{N}$, cf. [3, p. 150]. Now we apply Proposition 4.1. Condition 1) of Proposition 4.1 is fulfilled as a corollary of Proposition 6.2 or as a simple exercise using the multivariate CLT. Condition 2) of Proposition 4.1 follows from (13) with $a=b=2$ and $H=\sqrt{6} F$.

Lemma 6.2. Let $F$ be an arbitrary distribution function. The mapping $Q_{F}: D[0,1] \rightarrow D(\mathbf{R}): x \mapsto x \circ F$ (i.e., $Q_{F}(x)$ is the function $\left.t \mapsto x(F(t))\right)$ is $\mathscr{L}\left(B_{0}\right)$-a.e. continuous, where $\mathscr{L}\left(B_{0}\right)$ denotes the distribution of the Brownian bridge $B_{0}$.

P r o o f. We show that $Q_{F}$ is continuous as a function from $(C[0,1]$, $\|\cdot\|)$ to $D(\mathbf{R})$. This suffices because $\mathbf{P}\left(B_{0} \in C[0,1]\right)=1$ and the Skorokhod topology coincides with the uniform topology (the one induced by the supnorm $\|\cdot\|)$ on $C[0,1]$, cf. $\left[3\right.$, p. 124]. Now let $x_{n} \rightarrow x$ in $(C[0,1],\|\cdot\|)$. Since the image of $F$ is a subset of $[0,1]$, this implies

$$
\sup _{t \in \mathbf{R}}\left|x_{n}(F(t))-x(F(t))\right| \rightarrow 0 .
$$

Hence (5) is satisfied with $\lambda_{n} \equiv$ id (the identity function on $\mathbf{R}$ ) and we have $Q_{F}\left(x_{n}\right) \rightarrow Q_{F}(x)$ in $D(\mathbf{R})$.

Proposition 6.1. The series of random variables $\left\{\alpha_{n}^{F}\right\}$ in $(D, \mathscr{D})$ is tight for all distribution functions $F$.

P r o o f. The distribution function of the uniform $(0,1)$ distribution, which we denote by $G$, is continuous. Thus by Lemma 6.1 , Proposition 6.2 below, and Proposition 3.1 we have

$$
\alpha_{n}^{G} \stackrel{\mathscr{L}}{\longrightarrow} B_{1}^{G} \quad \text { in } \quad(D, \mathscr{D})
$$

If we restrict these processes to the time domain $[0,1]$, then the convergence remains true, cf. [3, p. 174, Theorem 16.7]. Furthermore, for any distribution function $F, \alpha_{n}^{F}$ and $B_{1}^{F}$ are their images under $Q_{F}$ (cf. Lemma 6.2). Hence the previous lemma allows us to apply the continuous mapping theorem: $\alpha_{n}^{F} \stackrel{\mathscr{L}}{\longrightarrow} B_{1}^{F} \quad$ in $(D, \mathscr{D})$. A convergent sequence is tight. 
Proposition 6.2. Let $\tau \in \mathbf{R}$, and let $F$ be an arbitrary distribution function such that Condition C.1 is satisfied. Then

$$
\begin{aligned}
& \left(\alpha_{n}^{F}\left(t_{1}\right), \ldots, \alpha_{n}^{F}\left(t_{k}\right), \beta_{n}^{F, \tau}\left(t_{1}\right), \ldots, \beta_{n}^{F, \tau}\left(t_{k}\right)\right) \\
& \quad \stackrel{\mathscr{L}}{\longrightarrow}\left(B_{1}^{F}\left(t_{1}\right), \ldots, B_{1}^{F}\left(t_{k}\right), N_{0}^{\varrho_{1}, \varrho_{2}}\left(t_{1}\right), \ldots, N_{0}^{\varrho_{1}, \varrho_{2}}\left(t_{k}\right)\right)
\end{aligned}
$$

holds true for all $k \in \mathbf{N}$ and $t_{1}, \ldots, t_{k} \in \mathbf{R}$.

A few remarks before we come to the proof: Showing $\alpha_{n} \stackrel{\mathscr{F}}{\longrightarrow} B_{1}$ is a straightforward application of the multivariate CLT. The result $\beta_{n} \stackrel{\mathscr{F}}{\longrightarrow} N_{0}$ is also easy to get using a Poisson-type limit theorem. Of course, it does not suffice to show these two statements separately. The two sequences are not independent of each other, and we need to show the finite-dimensional convergence of the joint sequence. Obviously neither of the approaches for the marginals works here. We prove Proposition 6.2 by showing the pointwise convergence of the corresponding characteristic functions. This includes some lengthy calculations, so we restrict our demonstration to

$$
\left(\alpha_{n}(t), \beta_{n}(t)\right) \stackrel{\mathscr{L}}{\longrightarrow}\left(B_{1}(t), N_{0}(t)\right),
$$

and only for $t \geqslant 0$. The case $t<0$ works just the same. The full-detail proof treating arbitrary tuples $t_{1}, \ldots, t_{k} \in \mathbf{R}$ is written down in [27]. For the characteristic function of $\left(\alpha_{n}(t), \beta_{n}(t)\right)$ we write $\psi_{n}^{(t)}$, or short $\psi_{n}$, and for the characteristic function of $\left(B_{1}(t), N_{0}(t)\right)$ we write $\psi^{(t)}$ or $\psi$. Since $B_{1}(t)$ and $N_{0}(t)$ are independent, we can write down, if $t \geqslant 0$,

$$
\psi^{(t)}(x, y)=\exp \left\{-\frac{1}{2} F(t)(1-F(t)) x^{2}+\varrho_{2} t\left(e^{i y}-1\right)\right\} .
$$

If $t<0$, then $\psi^{(t)}(x, y)$ contains a term with $\varrho_{1}$ instead of $\varrho_{2}$. The next lemma specifies $\psi_{n}^{(t)}$.

Lemma 6.3. Assume $t \geqslant 0$.

1) If $t>\tau$, and $n$ is sufficiently large such that $\tau+t / n<t$, then the characteristic function $\psi_{n}^{(t)}: \mathbf{R}^{2} \rightarrow \mathbf{C}$ of $\left(\alpha_{n}^{F}(t), \beta_{n}^{F, \tau}(t)\right)$ is given by

$$
\begin{aligned}
\psi_{n}^{(t)}(x, y)= & \exp \{-i x \sqrt{n} F(t)\}\left[1+F(t)\left(e^{i x / \sqrt{n}}-1\right)\right. \\
& \left.+\left(F\left(\tau+\frac{t}{n}\right)-F(\tau)\right)\left(e^{i y}-1\right) e^{i x / \sqrt{n}}\right]^{n} .
\end{aligned}
$$

2) If $t \leqslant \tau$, then $\psi_{n}^{(t)}: \mathbf{R}^{2} \rightarrow \mathbf{C}$ is

$$
\begin{aligned}
\psi_{n}^{(t)}(x, y)= & \exp \{-i x \sqrt{n} F(t)\}\left[1+F(t)\left(e^{i x / \sqrt{n}}-1\right)\right. \\
& \left.+\left(F\left(\tau+\frac{t}{n}\right)-F(\tau)\right)\left(e^{i y}-1\right)\right]^{n} .
\end{aligned}
$$


$\mathrm{P}$ r o o f. Keep in mind that $t \geqslant 0$. Per definition of the characteristic function,

$$
\psi_{n}(x, y)=\mathbf{E} \exp \left\{i x\left[\frac{1}{\sqrt{n}} \sum_{k=1}^{n}\left(\mathbb{I}_{(-\infty, t]}\left(X_{k}\right)-F(t)\right)\right]+i y \sum_{k=1}^{n} \mathbb{I}_{(\tau, \tau+t / n]}\left(X_{k}\right)\right\} .
$$

Since the $X_{k}, k=1, \ldots, n$, are i.i.d., this transforms to

$$
\left(\psi_{n}(x, y)\right)^{1 / n}=\mathbf{E} \exp \left\{\frac{i x}{\sqrt{n}}\left(\mathbb{I}_{(-\infty, t]}\left(X_{1}\right)-F(t)\right)+i y \mathbb{I}_{(\tau, \tau+t / n]}\left(X_{1}\right)\right\} .
$$

The right-hand side is the expectation of a function of the discrete random variable $\left(\mathbb{I}_{(-\infty, t]}\left(X_{1}\right), \mathbb{I}_{(\tau, \tau+t / n]}\left(X_{1}\right)\right)$, whose distribution is given in Table 1 .

Table 1.

\begin{tabular}{|c|c|c|}
\hline \multirow{2}{*}{$\left(\mathbb{I}_{(-\infty, t]}\left(X_{1}\right), \mathbb{I}_{\left(\tau, \tau+\frac{t}{n}\right]}\left(X_{1}\right)\right)$} & \multicolumn{2}{|c|}{ Corresponding probability if } \\
\cline { 2 - 3 } & $\tau+\frac{t}{n}<t$ & $t \leqslant \tau$ \\
\hline$(0,0)$ & $1-F(t)$ & $1-F\left(\tau+\frac{t}{n}\right)+F(\tau)-F(t)$ \\
$(0,1)$ & 0 & $F\left(\tau+\frac{t}{n}\right)-F(\tau)$ \\
$(1,0)$ & $F(t)-F\left(\tau+\frac{t}{n}\right)+F(\tau)$ & $F(t)$ \\
$(1,1)$ & $F\left(\tau+\frac{t}{n}\right)-F(\tau)$ & 0 \\
\hline
\end{tabular}

Thus in both cases we can write down the expectation (15) as a sum of three summands. Some re-grouping yields the expressions in Lemma 6.3.

$\mathrm{Pr}$ o of of Proposition 6.2. We show that $\psi_{n}^{(t)} \rightarrow \psi^{(t)}$ pointwise for all $t \geqslant 0$. We apply the following result from complex analysis. For complex numbers $c$ and $c_{n}, n \in \mathbf{N}$,

$$
c_{n} \rightarrow c \quad \Longrightarrow \quad\left(1+\frac{c_{n}}{n}\right)^{n} \rightarrow e^{c}
$$

Hence, it suffices to prove

$$
n\left(\psi_{n}^{1 / n}(x, y)-1\right) \longrightarrow \ln \psi(x, y) .
$$

Call the left-hand side $h_{n}$ and the right-hand side $h$. Consider at first the case of Lemma $6.3,1)$, i.e., $\tau<t$. Then by (14) and Lemma 6.3,

$$
\begin{aligned}
h_{n}= & n \exp \left\{-\frac{i x}{\sqrt{n}} F(t)\right\}\left[1+F(t)\left(e^{i x / \sqrt{n}}-1\right)\right. \\
& \left.+\left(F\left(\tau+\frac{t}{n}\right)-F(\tau)\right)\left(e^{i y}-1\right) e^{i x / \sqrt{n}}\right]-n, \\
h= & -\frac{1}{2} F(t)(1-F(t)) x^{2}+\varrho_{2} t\left(e^{i y}-1\right) .
\end{aligned}
$$

We break the convergence $h_{n} \rightarrow h$ down into two parts: 
(a) $n \exp \left\{-\frac{i x}{\sqrt{n}} F(t)\right\}\left[1+F(t)\left(e^{i x / \sqrt{n}}-1\right)\right]-n \longrightarrow-\frac{1}{2} F(t)(1-F(t)) x^{2}$,

(b) $n \exp \left\{\frac{i x}{\sqrt{n}}(1-F(t))\right\}\left[F\left(\tau+\frac{t}{n}\right)-F(\tau)\right]\left(e^{i y}-1\right) \longrightarrow \varrho_{2} t\left(e^{i y}-1\right)$.

Part (a). Use the Taylor expansion of the exponential function. Bear in mind that it converges uniformly on any compact set. This allows us to write

$$
\begin{aligned}
& \exp \left\{-\frac{i x F(t)}{\sqrt{n}}\right\}=1-\frac{i x F(t)}{\sqrt{n}}-\frac{x^{2} F(t)^{2}}{2 n}+o\left(\frac{1}{n}\right) \quad(n \rightarrow \infty), \\
& \exp \left\{\frac{i x}{\sqrt{n}}\right\}=1+\frac{i x}{\sqrt{n}}+o\left(\frac{1}{\sqrt{n}}\right) \quad(n \rightarrow \infty) .
\end{aligned}
$$

Plug this into the left-hand side, the rest is computing.

Part (b) becomes apparent by noting that

$$
\begin{aligned}
& \lim _{n} \frac{n}{t}\left(F\left(\tau+\frac{t}{n}\right)-F(\tau)\right)=\varrho_{2} \quad(t>0), \\
& \lim _{n} \exp \left\{\frac{i x}{\sqrt{n}}(1-F(t))\right\}=1 .
\end{aligned}
$$

By adding (a) and (b) we have proved $h_{n} \rightarrow h$. By (16) this implies $\psi_{n}^{(t)}(x, y) \rightarrow \psi^{(t)}(x, y)$, but so far only for $\tau<t$. As for $\tau \geqslant t$, the only difference is that in (b) the term $\exp \{(i x / \sqrt{n})(1-F(t))\}$ is replaced by $\exp \{-(i x / \sqrt{n}) F(t)\}$, which of course converges to 1 as well. Proposition 6.2 is proved.

7. Application in statistics. In this short section we demonstrate at an example how Theorem 2.1 can be useful in statistics. Our arguments will only briefly be sketched. Consider i.i.d. random variables $X_{1}, \ldots, X_{n}, n \in \mathbf{N}$, with values in $[0,1]$ and common distribution function $F=F_{\tau, \gamma}$. Here, $F_{\tau, \gamma}$ is defined on $[0,1]$ as the polygonal line through the points $(0,0),(\tau, \gamma)$, and $(1,1)$, where the parameters $\tau$ and $\gamma$ both lie in the open interval $(0,1)$, and it is assumed that $\tau \neq \gamma$. Thus, $\tau$ is the single point of discontinuity of the corresponding density. In this model Chernoff and Rubin [5] investigate the maximum likelihood estimator for $\tau$. An ad hoc estimator for the twodimensional parameter $(\tau, \gamma)$ is given by

$$
\widehat{\tau}_{n}=\underset{t \in[0,1]}{\arg \max }\left|\mathbb{F}_{n}(t)-t\right| \quad \text { and } \quad \widehat{\gamma}_{n}=\mathbb{F}_{n}\left(\widehat{\tau}_{n}\right) .
$$

A key role in the analysis of the pair

$$
\left(n\left(\widehat{\tau}_{n}-\tau\right), \sqrt{n}\left(\widehat{\gamma}_{n}-\gamma\right)\right)
$$

plays the observation that it has the same limit distribution as

$$
\left(\underset{t \in \mathbf{R}}{\arg \max }\left\{\operatorname{sign}(\gamma-\tau)\left(\beta_{n}^{F, \tau}(t)-t\right)\right\}, \alpha_{n}^{F}(\tau)\right) .
$$


Thus Theorem 2.1 and a (formal) application of the continuous mapping theorem yield convergence in distribution:

$$
\left(n\left(\widehat{\tau}_{n}-\tau\right), \sqrt{n}\left(\widehat{\gamma}_{n}-\gamma\right)\right) \stackrel{\mathscr{L}}{\longrightarrow}(A, B),
$$

where

$$
A=\underset{t \in \mathbf{R}}{\arg \max }\left\{\operatorname{sign}(\gamma-\tau)\left(N_{0}(t)-t\right)\right\}
$$

and $B \sim N[0, F(\tau)[1-F(\tau)])$ are independent. The rates of $N_{0}=N_{0}^{\varrho_{1}, \varrho_{2}}$ are given by $\varrho_{1}=\gamma / \tau$ and $\varrho_{2}=(1-\gamma) /(1-\tau)$. In [11] we give a representation of $A$ in terms of arrival times of $N_{0}$, which shows that with probability 1 the maximizing point $A$ is uniquely determined. Moreover $A$ is seen to have a continuous distribution function. A rigorous proof of (18) and further information will be published elsewhere.

\section{REFERENCES}

1. Al-Hussaini A., Elliott R. J. Convergence of the empirical distribution to the Poisson process. - Stochastics, 1984, v. 13, № 4, p. 299-308.

2. Bauer H. Maß- und Integrationstheorie. Berlin: de Gruyter, 1992, $260 \mathrm{p}$.

3. Billingsley P. Convergence of Probability Measures. New York: Wiley, 1999, 277 p.

4. Csörgö M., Horváth L. Convergence of the empirical and quantile distributions to Poisson measures. - Statist. Decis., 1988, v. 6, № 1-2, p. 129-136.

5. Chernoff H., Rubin H. The estimation of the location of a discontinuity in density. Proceedings of the Third Berkeley Symposium on Mathematical Statistics and Probability, v. I. Berkeley: Univ. of California Press, 1956, p. 19-37.

6. Donsker M. D. An invariance principle for certain probability limit theorems. - Mem. Amer. Math. Soc., 1951, v. 6, p. 1-12.

7. Donsker M.D. Justification and extension of Doob's heuristic approach to the Kolmogorov-Smirnov theorems. - Ann. Math. Statist., 1952, v. 23, p. 277-281.

8. Doob J. L. Heuristic approach to the Kolmogorov-Smirnov theorems. - Ann. Math. Statist., 1949, v. 20, p. 393-403.

9. Ethier S. N., Kurtz T. G. Markov Processes: Characterization and Convergence. New York: Wiley, 1986, $534 \mathrm{p}$.

10. Elstrodt J. Maß- und Integrationstheorie. Berlin: Springer-Verlag, 2005, $434 \mathrm{p}$.

11. Ferger $D$. On the minimizing point of the incorrectly centered empirical process and its limit distribution in nonregular experiments. - ESAIM Probab. Statist., 2005, v. 9 , p. $307-322$.

12. Галамбош Я. Асимптотическая теория экстремальных порядковых статистик. М.: Наука, 1984, 303 с.

13. Жакод Ж., Ширяев А.Н. Предельные теоремы для случайных процессов, т. 1, 2. М.: Наука, 1994, 542 с., 336 с.

14. Kabanov Yu. M., Liptser R. Sh., Shiryaev A.N. Some limit theorems for simple point processes (a martingale approach). — Stochastics, 1980, v. 3, № 3, p. 203-216.

15. Kolmogoroff A.N. Sulla determinazione empirica di una legge di distribuzione. Giorn. Ist. Ital. Attuari, 1933, v. 4, № 1, p. 83-91; рус. пер.: Об эмпирическом определении закона распределения. - А. Н. Колмогоров. Теория вероятностей и математическая статистика. М.: Наука, 1986, с. 134-141.

16. Колмогоров А.Н. О сходимости А.В.Скорохода. - Теория вероятн. и ее примен., 1956, т. 1 , в. 2, с. $239-247$. 
17. Lindvall $T$. Weak convergence of probability measures and random functions in the function space $D[0, \infty)$. - J. Appl. Probab., 1973, v. 10, p. 109-121.

18. Pollard D. Convergence of Stochastic Processes. New York: Springer-Verlag, 1984, $215 \mathrm{p}$.

19. Прохоров Ю. В. Сходимость случайных процессов и предельные теоремы теории вероятностей. - Теория вероятн. и ее примен., 1956, т. 1, в. 2, с. 177-238.

20. Revuz D., Yor M. Continuous Martingales and Brownian Motion. Berlin: SpringerVerlag, 1999, $602 \mathrm{p}$.

21. Rossberg H.-J. Über das asymptotische Verhalten der Rand- und Zentralglieder einer Variationsreihe. II. - Publ. Math. Debrecen, 1967, v. 14, p. 83-90.

22. Скороход A.В. Предельные теоремы для случайных процессов. - Теория вероятн. и ее примен., 1956, т. 1, в. 3, с. 289-319.

23. Смирнов Н.В. Приближение законов распределения случайных величин по эмпирическим данным. - Успехи матем. наук, 1944, в. 10, с. 179-206.

24. Stone C., Weak convergence of stochastic processes defined on semi-infinite time intervals. - Proc. Amer. Math. Soc., 1963, v. 14, p. 694-696.

25. Shorack G. R., Wellner J. A. Empirical Processes with Applications to Statistics. New York: Wiley, 1986, $939 \mathrm{p}$.

26. van der Vaart A. W., Wellner J. A. Weak Convergence and Empirical Processes. New York: Springer-Verlag, 1996, 508 p.

27. Vogel D. Weak convergence of the empirical process and the rescaled empirical distribution function in the Skorokhod product space. Master's thesis. Dresden: Technische Universität Dresden, 2005.

28. Whitt $W$. Weak convergence of probability measures on the function space $C[0, \infty)$. Ann. Math. Statist., 1970, v. 41, p. 939-944.

29. Whitt $W$. Weak Convergence of Probability Measures on the Function Space $D[0, \infty)$. Technical Report. Yale University, 1971.

30. Whitt W. Stochastic-Process Limits. New York: Springer-Verlag, 2002, 602 p.

Поступила в редакцию

30.XI.2008

Исправленный вариант

30.V.2009 